\title{
Testing for elliptical symmetry of errors in the multivariate linear regression model \\ Yan Su ${ }^{a}$, Shao-Yue Kang ${ }^{b}$
}

School of Mathematics and Physics, North China Electric Power University,

Baoding, 071003, China

aemail: suyanhd@163.com, bemail:kangsy5@163.com

Keywords: Linear model; Errors; Elliptical symmetry; Goodness-of-fit; Bootstrap approximation

\begin{abstract}
This paper presents a characteristic test for testing the elliptical distribution of the errors in the multivariate linear regression model. We obtain the asymptotic spherical distribution of the transformed residuals of the regression model under the null hypothesis. Based on bootstrap approximation, an algorithm is given to estimate the critical values of the test. The test statistic possesses symmetry and then the test power can be enhanced. The test is practical to implement for arbitrary dimension of the errors.
\end{abstract}

\section{Introduction}

A multivariate linear model describes the relationship between a response vector $y$ and a vector $x$ of covariables. Let $y_{1}, \cdots, y_{n}$ be $n$ independent observation vectors in $R^{m}$, following the model

$$
\begin{aligned}
& y_{j}^{\prime}=x_{j}^{\prime} \beta+\varepsilon_{j}^{\prime}, j=1, \cdots, \mathrm{n}, \\
& E\left(\varepsilon_{j}\right)=0, \operatorname{Cov}\left(\varepsilon_{j}\right)=\Sigma,
\end{aligned}
$$

where the prime "I" denotes transpose, the design vectors $x_{j} \in R^{p}$ are assumed to be nonrandom, $\beta$ is an unknown $p \times m$ matrix of parameters called regression coefficients, $\varepsilon_{j}, j=1, \cdots, n$ are the $m$ - vectors of errors, and $\Sigma$ is an unknown $m \times m$ positive definite matrix.

The family of elliptically symmetric distributions is a natural extension of the family of multivariate normal distributions. It contains short-tailed and long-tailed distributions, including symmetric Kotz type distributions, symmetric multivariate Pearson Types VII and II distributions. The errors in a multivariate regression model can be assumed to have an elliptical distribution when the normality assumption fails.

In this paper, we consider the multivariate linear model with errors $\varepsilon_{j}$ in (1)-(2) having an elliptical distribution. To avoid wrong conclusions in regression analysis, the distributional assumption on the errors should be checked. Let $F$ be the unknown distribution of the errors $\varepsilon_{j}$ and let $F_{0}$ be the elliptical distribution. We want to test the hypothesis

$$
F=F_{0} \text {. }
$$

Gamero, García and Mejías proposed a goodness-of-fit test for any fixed distribution of errors in multivariate linear models[1]. Su and Yang presented a goodness-of-fit test for uniformity on the surface of a unit sphere based on generalized inverse, the test possesses symmetry and has nice properties[2].

Let $\Omega_{m}$ denote the surface of a unit sphere centered at the origin in $R^{m}$ and let $U\left(\Omega_{m}\right)$ denote the uniform distribution on $\Omega_{m}$. Let $\hat{\varepsilon}_{j}$ be the residuals of the multivariate linear regression model. The asymptotic null distribution of the transformed residuals is a spherical distribution. Based on a simple property for the spherical distribution, the goodness-of-fit test for the elliptical distribution of the errors $\varepsilon_{j}$ in (1) can be translated into the goodness-of-fit test for $U\left(\Omega_{m}\right)$. We introduce a characterization-based test for the elliptical distribution of the errors $\varepsilon_{j}$ in (1). The transformation 
based on Cholesky decomposition leads to the transformed residuals whose joint distribution asymptotically does not depend on the unknown matrix $\Sigma_{m}$ of the elliptical distribution. Hence, the critical values can be approximated by Monte Carlo and bootstrap samples.

The paper is organized as follows. In Section 2, we introduce the multivariate linear regression model and some lemmas. In Section 3, the generalized inverse-based test for the elliptical distribution of the errors is proposed. The asymptotic null spherical distribution of the transformed residuals is obtained. In Section 4, the algorithm to estimate the critical values is given. The conclusion and a possible extension of the obtained results are present in Section 5. The proofs of Theorem1 and Lemma2(b). are postponed to Appendix.

\section{The multivariate linear model and some lemmas}

Definition $^{[3]}$ Let $U^{(\mathrm{m})} \sim U\left(\Omega_{m}\right)$. An $m \times 1$ random vector $\varsigma$ is said to have a spherical distribution if $\varsigma$ has a stochastic representation $\varsigma^{d}=\kappa \cdot U^{(\mathrm{m})}$ for some random variable $\kappa \geq 0$, which is independent of $U^{(\mathrm{m})}$. Here $\stackrel{d}{=}$ signifies that the two sides have the same distribution.

Definition $2^{[3]}$ Let $A^{\prime}$ be an $m \times m$ matrix of rank $m$ and $\Sigma_{m}=A^{\prime} A$. An $m \times 1$ random vector $\eta$ is said to have an elliptical distribution with parameters $\mu(m \times 1)$ and $\Sigma_{m}$ if

$$
\eta \stackrel{d}{=} \mu+\kappa A^{\prime} U^{(d)}
$$

where random variable $\kappa \geq 0$ is independent of $U^{(\mathrm{m})}$. We shall use the notation $\eta \sim E C_{m}\left(\mu, \Sigma_{m}\right)$. Let $I_{n}$ denotes the $n \times n$ identity matrix and let

$$
Y=\left(y_{1}, \cdots, y_{n}\right)^{\prime}, X=\left(x_{1}, \cdots, x_{n}\right)^{\prime}, \varepsilon=\left(\varepsilon_{1}, \cdots, \varepsilon_{n}\right)^{\prime}
$$

Then the multivariate linear model (1)-(2) takes the form

$$
\begin{gathered}
Y=X \beta+\varepsilon, \\
E\left[\operatorname{vec}\left(\varepsilon^{\prime}\right)\right]=0, \operatorname{Cov}\left[\operatorname{vec}\left(\varepsilon^{\prime}\right)\right]=I_{n} \otimes \Sigma,
\end{gathered}
$$

where $Y$ and $\varepsilon$ are $n \times m$ random matrices, $X$ is a known $n \times p$ matrix, and $\beta$ is an unknown $p \times m$ matrix. Here, the $\operatorname{sign} \otimes$ denotes the kronecker product of matrices.

The multivariate linear model (6) -(7) generalizes the multiple linear model $(m=1)$ by allowing a vector of observations, given by the rows of a matrix $Y$, to correspond to the rows of the design matrix $X$.

Lemma1 $^{[3]}$ Assume that $\eta \sim E C_{m}\left(\mu, \Sigma_{m}\right)$ with $E\left(\kappa^{2}\right)<\infty$, where $\kappa$ is defined in (4). Let $\operatorname{Cov}(\eta)$ denotes the covariance matrix of $\eta$. Then $\operatorname{Cov}(\eta)$ exists and

$$
E(\eta)=\mu, \operatorname{Cov}(\eta)=\Sigma=\frac{E\left(\kappa^{2}\right)}{m} \Sigma_{m} .
$$

Lemma2 Let the model $Y=X \beta+\varepsilon$ be defined in (6). Let $\varepsilon_{1}, \cdots, \varepsilon_{n}$ be i.i.d. $\sim E C_{m}\left(0, \Sigma_{m}\right)$, where $\varepsilon_{1}, \cdots, \varepsilon_{n}$ are defined by (5) and $\operatorname{Cov}\left(\varepsilon_{1}\right)=\Sigma$ exists. Let $\operatorname{rank}(X)=p$ and let

$$
P_{X}=X\left(X^{\prime} X\right)^{-1} X^{\prime} \text {. }
$$

Let $\hat{\beta}$ be the least squares estimate of $\beta$, i.e., $\hat{\beta}=\left(X^{\prime} X\right)^{-1} X^{\prime} Y$. Let

$$
\hat{\varepsilon}=\left(\hat{\varepsilon}_{1}, \cdots \hat{\varepsilon}_{n}\right)^{\prime}=Y-X \hat{\beta}, \quad \hat{\Sigma}=\frac{1}{n-p} \hat{\varepsilon}^{\prime} \hat{\varepsilon}, \lim _{n \rightarrow \infty} \frac{1}{n} X^{\prime} X=D,
$$

where $D$ is a positive definite matrix. Then (a). ${ }^{[4]}$

$$
\hat{\varepsilon}=\left(\hat{\varepsilon}_{1}, \cdots, \hat{\varepsilon}_{n}\right)^{\prime}=\left(I_{n}-P_{X}\right) \varepsilon, \quad \hat{\beta} \stackrel{P}{\rightarrow} \beta, n \rightarrow \infty,
$$

where $\stackrel{P}{\rightarrow}$ denotes convergence in probability as $n \rightarrow \infty$. 
(b).

$$
\hat{\Sigma} \stackrel{P}{\rightarrow} \Sigma, n \rightarrow \infty,
$$

Lemma3 $^{[3]}$ Assume that $\varphi \sim E C_{m}\left(0, \Sigma_{m}\right)$ with $\operatorname{Rank}\left(\Sigma_{m}\right)=m, B$ is an $m \times m$ matrix. Then

$$
B^{\prime} \varphi \sim E C_{m}\left(0, B^{\prime} \Sigma_{m} B\right) \text {. }
$$

Lemma4 $^{[5]}$ Let $\varepsilon_{i}, i \leq n$ be defined in (5). Let $\varepsilon_{1}, \cdots, \varepsilon_{n}$ be i.i.d. $\sim E C_{m}\left(0, \Sigma_{m}\right)$ and let $\tilde{\Sigma}=\varepsilon^{\prime} \varepsilon /(n-p)$. Let the Cholesky decomposition of $\tilde{\Sigma}$ be $\tilde{\Sigma}=[L(\tilde{\Sigma})][L(\tilde{\Sigma})]^{\prime}$, where $L(\tilde{\Sigma})$ is lower triangular with positive diagonal elements . Let

$$
w_{i}=[L(\tilde{\Sigma})]^{-1} \varepsilon_{i}, i=1, \cdots, \mathrm{n}, \quad W=\left(w_{1}, \cdots, w_{n}\right)^{\prime} .
$$

Then the distribution of $W$ does not depend on $\Sigma_{m}$.

Lemma $^{[3]}$ If An $m \times 1$ random vector $\varsigma$ has a spherical distribution then

$$
\varsigma /\|\varsigma\| \sim U\left(\Omega_{m}\right),
$$

where $\|\cdot\|$ denotes the Euclidean norm.

Lemma6 ${ }^{[2]}$ Let $U^{(\mathrm{m})}=\left(U_{1}, \cdots, U_{m}\right)^{\prime} \sim U\left(\Omega_{m}\right)$ and let $G^{(\mathrm{m})}=\left(U_{1}^{2}, \cdots, U_{m}^{2}\right)^{\prime}, \mu^{(\mathrm{m})}=(1 / m, \cdots, 1 / m)^{\prime}$. Let $U_{i}^{(\mathrm{m})}=\left(U_{1 i}, \cdots, U_{m i}\right)^{\prime}, i=1, \cdots, n$ be i.i.d. $\sim U\left(\Omega_{m}\right)$ and let

$$
Q_{j n}=\frac{1}{n} \sum_{i=1}^{n} U_{j i}^{2}, j=1, \cdots, m, \quad V_{n}^{(\mathrm{m})}=\left(Q_{1 n}, \cdots, Q_{m n}\right)^{\prime} .
$$

Then

(a). The covariance matrix of $G^{(\mathrm{m})}$ is an $m \times m$ matrix $\sigma^{2} \cdot\left(a_{i j}\right)=\sigma^{2} M$ with

$$
\begin{gathered}
\sigma^{2}=\frac{2}{m^{2}(m+2)}, M=\left(a_{i j}\right), \\
a_{i i}=m-1, \quad i=1, \cdots, m, \quad a_{i j}=-1, \quad i, j=1, \cdots, m, i \neq j .
\end{gathered}
$$

(b). $\operatorname{rank}(M)=m-1$ and $M^{+}=\left(1 / m^{2}\right) M$.

(c).

$$
\begin{gathered}
R_{n}=\sqrt{n}\left(V_{n}^{(\mathrm{m})}-\mu^{(\mathrm{m})}\right) \stackrel{d}{\rightarrow} N_{m}\left(0, \sigma^{2} M\right) \\
R_{n}^{\prime} \sigma^{-2} M^{-} R_{n} \stackrel{d}{\rightarrow} \chi_{m-1}^{2}, \quad n \rightarrow \infty, \gamma=R_{n}^{\prime}(\sigma m)^{-2} M R_{n} \stackrel{d}{\rightarrow} \chi_{m-1}^{2}, n \rightarrow \infty,
\end{gathered}
$$

where $\stackrel{d}{\rightarrow}$ denotes convergence in distribution as $n \rightarrow \infty, \chi_{m-1}^{2}$ is the chi-squared distribution with $d-1$ degrees of freedom.

Remark1 $\operatorname{Cov}\left(U^{(m)}\right)=m^{-1} I_{m}$ corresponds to the moment of inertia of $U^{(m)} \sim U\left(\Omega_{m}\right)$. Consider a system of $n$ particles on $\Omega_{m}$ with unit mass. If the $n$ particles are uniformly distributed on $\Omega_{m}$, then the moment of inertia of the system about arbitrary unit vector should be nearly the same.

\section{Goodness of fit test for the elliptical distribution of errors}

Let $\Sigma$ and $\hat{\Sigma}$ be defined in (7) and (9), respectively. Let the Cholesky decomposition of $\Sigma, \hat{\Sigma}$ and $\Sigma_{m}$ be

$$
\Sigma=[L(\Sigma)][L(\Sigma)]^{\prime}, \hat{\Sigma}=[L(\hat{\Sigma})][L(\hat{\Sigma})]^{\prime}, \Sigma_{m}=\left[L\left(\Sigma_{m}\right)\right]\left[L\left(\Sigma_{m}\right)\right]^{\prime},
$$

respectively. Let $L^{-1}$ be the inverse of $L$ and let $\hat{\varepsilon}_{i}$ be defined in (9). Let

$$
\begin{gathered}
z_{i}=[L(\hat{\Sigma})]^{-1} \hat{\varepsilon}_{i}, i=1, \cdots, \mathrm{n}, \quad Z=\left(z_{1}, \cdots, z_{n}\right)^{\prime}, \\
\xi_{i}^{(\mathrm{m})}=z_{i} /\left\|z_{i}\right\|=\left(\xi_{1 i}, \cdots, \xi_{m i}\right)^{\prime}, i=1, \cdots, n, \quad \psi^{(\mathrm{m})}=\left(\xi_{1}^{(\mathrm{m})}, \cdots, \xi_{n}^{(\mathrm{m})}\right)^{\prime} .
\end{gathered}
$$

Theorem1 Let the conditions of Lemma2 hold. Let the $n \times m$ matrix $Z$ and the $m$-vectors $\xi_{i}^{(\mathrm{m})}, i \leq n$ be defined in (17) and (18), respectively. Let $\alpha^{2}=m^{-1} E(\kappa)$ with $\alpha>0$, where $\kappa$ is defined in (4). Then 
(a). The asymptotic distribution of $z_{i}$ is $E C_{m}\left(0, \alpha^{-2} I_{m}\right)$, which we write as $Z_{i} \stackrel{a}{\sim} E C_{m}\left(0, \alpha^{-2} I_{m}\right), i=1, \cdots, n$.

(b). $z_{1}, \cdots, z_{n}$ are asymptotically independent and the distribution of $Z$ asymptotically does not depend on the parameter $\Sigma_{m}$ of $E C_{m}\left(0, \Sigma_{m}\right)$.

(c). The asymptotic distribution of $\xi_{i}^{(\mathrm{m})}$ is $U\left(\Omega_{m}\right)$ and $\xi_{1}^{(\mathrm{m})}, \cdots, \xi_{n}^{(\mathrm{m})}$ are asymptotically independent.

Let $\left.\xi_{i}^{(\mathrm{m})}=\left(\xi_{1 i}, \cdots, \xi_{m i}\right)\right)^{\prime}$ be defined in (18) and let $\sigma^{2}$ and $M$ are defined in (14), respectively. Let

$$
\begin{aligned}
& \tilde{Q}_{j n}=\frac{1}{n} \sum_{i=1}^{n} \xi_{j i}^{2}, j=1, \cdots, m, \quad \tilde{V}_{n}^{(\mathrm{m})}=\left(\tilde{Q}_{1 n}, \cdots, \tilde{Q}_{m n}\right)^{\prime}, \\
& \tilde{R}_{n}=\sqrt{n}\left(\tilde{V}_{n}^{(\mathrm{m})}-\mu^{(\mathrm{m})}\right), \quad T=T(\hat{\varepsilon})=\tilde{R}_{n}^{\prime}(\sigma m)^{-2} M \tilde{R}_{n},
\end{aligned}
$$

where $\mu^{(m)}$ is defined in (15).

Remark2 Consider the null hypothesis (3), where $F_{0}$ denotes the $E C_{m}\left(0, \Sigma_{m}\right)$ distribution with the parameter $\Sigma_{m}$ unknown. By Theorem1, the goodness-of-fit test for $F_{0}$ can be translated into the goodness-of-fit test for $\xi_{i}^{(\mathrm{m})} \stackrel{a}{\sim} U\left(\Omega_{m}\right), i=1, \cdots, n$. By Lemma6(c), the elliptical symmetry is rejected for large values of $T(\hat{\varepsilon})$ in (20).

\section{The algorithm to implement the test statistic}

The algorithm to compute the test statistic.

The algorithm to compute $T(\hat{\varepsilon})$ in (20) consists of the following steps:

1. Compute the values of $\hat{\varepsilon}$ and $\hat{\Sigma}$ in (9), respectively.

2. Compute the value of $Z$ in (17).

3. Compute the value of $\psi^{(\mathrm{m})}$ in (18).

4. Compute the value of $\tilde{V}_{n}^{(\mathrm{m})}$ in (19).

5. Compute the values of $\tilde{R}_{n}$ and $T(\hat{\varepsilon})$ in (20), respectively.

The elliptical symmetry is rejected for large value of $T(\hat{\varepsilon})$.

The algorithm to estimate the critical values.

Let $z_{i}$ be defined in (17) and let

$$
\tau_{i}=\left\|z_{i}\right\|, i=1, \cdots, n,
$$

where $\|\cdot\|$ denotes the Euclidean norm. The bootstrap method uses the empirical distribution of $\tau_{i}, i=1, \cdots, n$ to approximate the distribution of $\kappa$ in (4).

The algorithm to estimate the critical values of $T(\hat{\varepsilon})$ consists of the following steps:

1. Sample $\tau_{1}^{*}, \cdots, \tau_{n}^{*}$ with replacement from the values $\tau_{1}, \cdots, \tau_{n}$.

2. Generate $U_{1}^{*}, \cdots, U_{n}^{*}$ which are i.i.d. uniform on $\Omega_{m}$. Compute $\varepsilon_{i}^{*}=\tau_{i}^{*} U_{i}^{*}, i=1, \cdots, n$.

3. Let $\varepsilon^{*}=\left(\varepsilon_{1}^{*}, \cdots, \varepsilon_{n}^{*}\right)^{\prime}$. Compute $\hat{\varepsilon}^{*}=\left(\hat{\varepsilon}_{1}^{*}, \cdots, \hat{\varepsilon}_{n}^{*}\right)^{\prime}=\left(I_{n}-P_{X}\right) \varepsilon^{*}$, where $P_{X}$ is defined in (8).

4. Compute $\hat{\Sigma}^{*}=\left[\hat{\varepsilon}^{*}\right]^{\prime} \hat{\varepsilon}^{*} /(n-p), z_{i}^{*}=\left[L\left(\hat{\Sigma}^{*}\right)\right]^{-1} \hat{\varepsilon}_{i}^{*}, i=1, \cdots, \mathrm{n}$, where $\hat{\Sigma}^{*}=\left[L\left(\hat{\Sigma}^{*}\right)\right]\left[L\left(\hat{\Sigma}^{*}\right)\right]^{\prime}$ ( the Cholesky decomposition).

5. Compute $\xi_{i}^{*}=z_{i}^{*} /\left\|z_{i}^{*}\right\|=\left(\xi_{1 i}^{*}, \cdots, \xi_{m i}^{*}\right)^{\prime}, i=1, \cdots, n$.

6. Compute $\tilde{Q}_{j n}^{*}=\frac{1}{n} \sum_{i=1}^{n}\left[\xi_{j i}^{*}\right]^{2}, j=1, \cdots, m, \tilde{V}_{n}^{*}=\left(\tilde{Q}_{1 n}^{*}, \cdots, \tilde{Q}_{m n}^{*}\right)^{\prime}$. 
7. Compute $\tilde{R}_{n}^{*}=\sqrt{n}\left(\tilde{V}_{n}^{*}-\mu^{(\mathrm{m})}\right)$, where $\mu^{(\mathrm{m})}=(1 / m, \cdots, 1 / m)^{\prime}$.

8. Compute $T^{*}=T\left(\hat{\varepsilon}^{*}\right)=\left[\tilde{R}_{n}^{*}\right]^{\prime}(\sigma m)^{-2} M \tilde{R}_{n}^{*}$, where $\sigma^{2}$ and $M$ are defined in (14), respectively.

Doing these $N$ times gives a sample of replicates $T_{1}^{*}, \cdots, T_{N}^{*}$. Let $T_{(1)}^{*}, \cdots, T_{(N)}^{*}$ be the order statistics, the critical values for $T(\hat{\varepsilon})$ can be estimated from $T_{(1)}^{*}, \cdots, T_{(N)}^{*}$.

\section{Conclusions}

Elliptical distribution plays an important role in generalized multivariate analysis. When the distribution of the errors $\varepsilon_{j}, j=1, \cdots, n$ in (1) enjoys elliptical symmetry, the direction vectors $\xi_{i}^{(\mathrm{m})}$ in (18) should be, approximately, uniformly distributed on the surface of the unit sphere $\Omega_{m}$. Based on the generalized inverse of the covariance matrix $\sigma^{2} M$ of $G^{(\mathrm{m})}$ in Lemma6, $T(\hat{\varepsilon})$ in (20) is constructed which possesses symmetry. Hence, the proposed test statistic $T(\hat{\varepsilon})$ will have good power for testing goodness of fit to the elliptical distribution of errors in the multivariate linear model.

The multivariate times series $\zeta_{t}$ follows a vector autoregressive(VAR) model, if

$$
\zeta_{t}=\phi_{0}+\sum_{i=1}^{p} \phi_{i} \zeta_{t-i}+a_{t}
$$

where $a_{t}$ is a sequence of i.i.d. random vectors with mean zero and covariance matrix $\Sigma_{a}$. The goodness-of-fit test for the elliptical distribution of the errors $\varepsilon_{t}$ in the multivariate linear regression model can be extended to testing the elliptical distribution of the innovations $a_{t}$ in the VAR model.

\section{Appendix}

Proof of Lemma2(b). By (a), we have

$$
\begin{aligned}
\hat{\Sigma} & =\frac{1}{n-p} \hat{\varepsilon}^{\prime} \hat{\varepsilon}=\frac{1}{n-p} \varepsilon^{\prime}\left(I_{n}-X\left(X^{\prime} X\right)^{-1} X^{\prime}\right) \varepsilon \\
& =\frac{n}{n-p}\left[\frac{1}{n} \varepsilon^{\prime} \varepsilon-\left(\frac{1}{n} \varepsilon^{\prime} X\right)\left(\frac{1}{n} X^{\prime} X\right)^{-1}\left(\frac{1}{n} X^{\prime} \varepsilon\right)\right] .
\end{aligned}
$$

By Lemma1 and the law of large numbers,

$$
\frac{1}{n} \varepsilon^{\prime} \varepsilon \stackrel{p}{\rightarrow} \frac{E\left(\kappa^{2}\right)}{m} \cdot \Sigma_{m}=\Sigma=\left(\sigma_{i j}\right)_{m \times m}, \quad n \rightarrow \infty .
$$

Let $X=\left(X_{(1)}, \cdots, X_{(p)}\right), \varepsilon=\left(\varepsilon_{(1)}, \cdots, \varepsilon_{(m)}\right)$ and $X^{\prime} \varepsilon=\left(\lambda_{i j}\right)_{p \times m}$. Then

$$
\lambda_{i j}=X_{(i)}^{\prime} \varepsilon_{(j)}, i=1, \cdots, p, j=1, \cdots, m \text {. }
$$

By Chebyshev's inequality for any $\Delta>0$,

$$
P\left(\left|n^{-1} \lambda_{i j}\right|>\Delta\right) \leq \frac{1}{n^{2} \Delta^{2}} \operatorname{Cov}\left(X_{(i)}^{\prime} \varepsilon_{(j)}\right)=\frac{X_{(i)}^{\prime} X_{(i)}}{n \Delta^{2}} \cdot \frac{\sigma_{j j}}{n},
$$

where $\sigma_{j j}>0$ is defined in (23). By (9), we have

$$
n^{-1} \lambda_{i j} \stackrel{p}{\rightarrow} 0, n^{-1} \cdot X^{\prime} \dot{\varepsilon} \stackrel{p}{\rightarrow} 0, \quad n \rightarrow \infty,
$$

By (23) and (25), $\quad \hat{\Sigma} \stackrel{p}{\rightarrow} \Sigma, \quad n \rightarrow \infty$.

Proof of Theorem1. By (9) and Lemma2(a),

$$
\hat{\varepsilon}_{i}^{\prime}=y_{i}^{\prime}-x_{i}^{\prime} \hat{\beta} \stackrel{p}{\rightarrow} \varepsilon_{i}^{\prime}, n \rightarrow \infty .
$$

Thus, the asymptotic distribution of $\hat{\varepsilon}_{i}$ is $E C_{m}\left(0, \Sigma_{m}\right)$, i.e., $\quad \hat{\varepsilon}_{i}^{a} \sim E C_{m}\left(0, \Sigma_{m}\right), i=1,2, \cdots, n$. 
By Lemma1 and (16), we have $\Sigma=\alpha^{2} \Sigma_{m}$. By Lemma2(b),

$$
L(\hat{\Sigma}) \stackrel{P}{\rightarrow} L(\Sigma)=\alpha L\left(\Sigma_{m}\right), n \rightarrow \infty
$$

Thus,

$$
z_{i}=[L(\hat{\Sigma})]^{-1} \hat{\varepsilon}_{i} \stackrel{P}{\rightarrow} \tilde{z}_{i}=\left[\alpha L\left(\Sigma_{m}\right)\right]^{-1} \varepsilon_{i}, n \rightarrow \infty,
$$

where $\varepsilon_{i}$ is defined in (5). Since $\varepsilon_{i} \sim E_{m}\left(0, \Sigma_{m}\right)$, by (27) - (28) and Lemma3, we have

$$
\tilde{z}_{i} \sim E C_{m}\left(0, \alpha^{-2} I_{m}\right), \quad z_{i} \stackrel{a}{\sim} E C_{m}\left(0, \alpha^{-2} I_{m}\right),
$$

Thus, the desired results of (a) and (b) are proved. Since $\tilde{z}_{i}$ in (29) has a spherical distribution, we have by Lemma5

$$
\xi_{i}^{(\mathrm{m})} \stackrel{d}{\rightarrow} \tilde{z}_{i} /\left\|\tilde{z}_{i}\right\| \sim U\left(\Omega_{m}\right), n \rightarrow \infty .
$$

The desired result of (c) is obtained.

\section{References}

[1] M.D.J. Gamero, J.M. García and R.P. Mejías: Testing goodness of fit for the distribution of errors in multivariate linear models. Journal of multivariate analysis, 95(2005), p. 301 322.

[2] Y. Su, Z.H. Yang: Goodness-of-fit test for uniformity on the surface of a unit sphere based on generalized inverse. In : Recent Advance in Statistics Application and Related Areas (Conference Proceedings of The 4th International Institute of Statistics \& Management Engineering Symposium, Dalian, China), edited by K.L. Zhu, H. Zhang, Aussino Academic Publishing House, Part 2, Sydney, (2011) p.1323 1327.

[3] K.T. Fang, S. Kotz and K.W. Ng: Symmetric Multivariate and Related Distributions. Chapman \& Hall, London, New York (1990).

[4] W.H. Greene: Economrtric Analysis, 4th ed. Prentice Hall, Inc.(2000).

[5] F.W. Huffer, C. Park: A test for elliptical symmetry. Journal of multivariate analysis, 98 (2007), p. 256 281.

[6] R.J. Muirhead: Aspects of multivariate statistical theory. John Wiley \& Sons, Inc., New York Chichester - Brisbane - Toronto - Singapore (1982).

[7] J.A. Díaz-García, R. Gutiérrez-Jáimez: The distribution of the residual from a general elliptical multivariate linear model. Journal of Multivariate Analysis, 97 (2006), p.1829 1841.

[8] B.M.G. Kibria, Haq M.S. Haq: Predictive inference for the elliptical linear model. Journal of Multivariate Analysis, 68 (1999), p. 235-249.

[9] L.X. Zhu, R.Q. Zhu and S.Song: Diagnostic checking for multivariate regression models. Journal of Multivariate Analysis, 99(2008), p.1841 1859.

[10] J.D. Hamilton: Time series analysis, Princeton University Press(1994).

[11] S.Pynnönen: Distribution of an arbitrary linear transformation of internally studentized residuals of multivariate regression with elliptical errors. Journal of Multivariate Analysis, 107 (2012), p. 40 52.

[12] R.S. Tsay: Multivariate time series analysis with R and financial applications. John Wiley \& Sons, Inc. Hoboken, New Jersey (2014). 